tries then were refined at B3LYP/6-311+ $\mathrm{G}^{* *}$. Correlated ab initio methods $\left(\operatorname{CCSD}(\mathrm{T}) / 6-311+\mathrm{G}^{* *}\right.$ for 1 to 3, MP2/6-31G* and MP2/6-311+G** for 1 to 6 ) were employed to reoptimize some of the geometries as further checks. Frequency calculations at MP2/6$311+\mathrm{G}^{* *}$ for $\mathbf{1}$ to $\mathbf{3}$ and MP2/6-31G* for $\mathbf{1}$ to $\mathbf{6}$ further confirm they are minima [Web fig. 2 (3)]. All calculations were carried out using Gaussian G98 (M. J. Frisch et al., Gaussian, Pittsburgh, PA, 1998).

5. Typical reference bond length at $B 3 L Y P / 6-311+G^{* *}$ are: $\mathrm{r}_{\mathrm{CC}}=1.531 \AA$ in ethane, $1.396 \AA$ in benzene, and $1.329 \AA$ in ethene; $r_{C B}=1.554 \AA$ in $\mathrm{CH}_{3} \mathrm{BH}_{2}$ and $1.396 \AA$ in $\mathrm{CH}_{2} \mathrm{BH}$; and $\mathrm{r}_{\mathrm{BB}}=1.629 \AA$ in $\mathrm{D}_{2 \mathrm{~d}} \mathrm{H}_{2} \mathrm{BBH}_{2}$.

6. P. v. R. Schleyer et al., J. Am. Chem. Soc. 118,6317 (1996). An extended bibliography of NICS is given by S. Patchkovskii and W. Thiel [J. Mol. Model. 6, 67 (2000)]

7. The data in Table 1 confirm (i) the expected $\pi$ electron counts $\left(\pi_{\text {tot }}\right)$ from natural bond orbital (NBO) analysis [A. E. Reed, L. A. Curtiss, F. Weinhold, Chem. Rev. 88, 899 (1988)]; (ii) that the $\pi$ occupancy increments $(\Delta \pi)$ contributed by $\mathbf{A}, \mathbf{B}$, and $\mathbf{C}$ are close to two, one, and zero electrons, respectively; (iii) that the NICS(1) values are consistent with the Hückel rule [compounds with $4 n+2 \pi$ electrons have negative $\operatorname{NICS}(1)$, whereas those with $4 n \pi$ electrons have positive values]; and (iv) the $\mathrm{H}$ chemical shifts in aromatic compounds are deshielded [ $\delta \mathrm{Hs}>7.0$ parts per million ( $\mathrm{ppm})]$, whereas those in antiaromatic compounds are shielded ( $\delta \mathrm{Hs}<7.0 \mathrm{ppm})$

8. The $\mathrm{WBI}$ is a measure of the bond order based on natural bond orbital analysis. The individual indices for each of the five bonds to the central carbons varies, but the total WBls to the ppCs for $\mathbf{4}, \mathbf{5}$, and $\mathbf{6}$ as well as other hyparenes, $\mathbf{7}$ and $\mathbf{8}$ are close to 4 ( Table 1).

9. K. Exner, P. v. R. Schleyer, Science 290, 1937 (2000).

10. Planar tetracoordinate carbon is increasingly well represented. For the latest examples and literature references, see Z.-X. Wang, P. v. R. Schleyer, J. Am. Chem. Soc. 123, 994 (2001); Z.-X. Wang et al., Org. Lett. 3, 9 (2001).

11. $\mathrm{SiB}_{8}\left(D_{8 \mathrm{~h}}\right)$ with a planar silicon, as well as $\mathrm{B}_{9}{ }^{-}\left(\mathrm{D}_{8 \mathrm{~h}}\right)$ and $\mathrm{CB}_{8}\left(\mathrm{C}_{2 \mathrm{v}}\right)$ with a boron in the center are minima.

12. G. A. Olah et al., Hypercarbon Chemistry (John Wiley, \& Sons, New York, 1987).

13. NICS(1) describes the NICS value $1 \AA$ above a ring center, where the $\pi$ ring current effects dominate over the local $\sigma$ contributions [P. v. R. Schleyer et al., J. Am. Chem. Soc. 119, 12669 (1997)].

14. In the following discussion, the NICS(1) and proton chemical shifts $(\delta \mathrm{H})$, shown in Table 1 , are used as criteria to judge aromaticity and antiaromaticity.

15. P. v. R. Schleyer, K. Najafian, Inorg. Chem. 37, 3455 (1998), and references therein.

16. S. Becker, H.-J. Dietze, Int. J. Mass Spectrom. Ion Processes 82, 287 (1988).

17. Computed vibrational spectra and NMR chemical shifts can be obtained from the authors on request. We will gladly cooperate with experimentalists.

18. F. M. Ge et al, Chem. J. Chinese Univ. 17, 1458 (2001)

19. F. M. Ge et al., Chem. J. Chinese Univ. 18, 1838 (2001).

20. J. Nagamatsu et al., Nature 410, 63 (2001).

21. The $\mathrm{D}_{5 \mathrm{~h}} \mathrm{CSi}_{5}{ }^{2-}$ dianion and its isoelectronic analogs, $\mathrm{C}_{2 v} \mathrm{CSi}_{4} \mathrm{P}^{-}$and $\mathrm{C}_{2 v} \mathrm{CSi}_{3} \mathrm{P}_{2}$, are minima with $\mathrm{PPCs}$ at B3LYP/6-311+G**//B3LYP/6-311+G** (P. V. R. Schleyer, K. Exner, unpublished data).

22. The closest analogy is the transition metal planar tetracoordinate carbon complex [see S. L. Buchwald et al., J. Am. Chem. Soc. 111, 397 (1989)].

23. D. Röttger, G. Erker, Angew. Chem. Int. Ed. Engl. 36, 812 (1997).

24. W. Siebert, A. Gunale, Chem. Soc. Rev. 28, 367 (1999).

25. For example, replacing two $-(\mathrm{CH})_{3}-$ subunits in cyclooctatetraene by two $-\mathrm{C}_{3} \mathrm{~B}_{2}-$ groping gives a $\mathrm{C}_{8} \mathrm{H}_{2} \mathrm{~B}_{4}$ compound with two planar tetracoordinate carbon atoms.

26. We dedicate this paper to G. A. Olah for his contributions to hypercarbon chemistry. Z.X.W. thanks R.-Z. Liu and M.-B. Huang for their encouragement.

R E P O R T S

\title{
Predicting the Mesophases of Copolymer-Nanoparticle Composites
}

\author{
Russell B. Thompson, ${ }^{1}$ Valeriy V. Ginzburg, ${ }^{1 *}$ Mark W. Matsen, ${ }^{2}$ \\ Anna C. Balazs ${ }^{1} \dagger$
}

The interactions between mesophase-forming copolymers and nanoscopic particles can lead to highly organized hybrid materials. The morphology of such composites depends not only on the characteristics of the copolymers, but also on the features of the nanoparticles. To explore this vast parameter space and predict the mesophases of the hybrids, we have developed a mean field theory for mixtures of soft, flexible chains and hard spheres. Applied to diblocknanoparticle mixtures, the theory predicts ordered phases where particles and diblocks self-assemble into spatially periodic structures. The method can be applied to other copolymer-particle mixtures and can be used to design novel composite architectures.

Mixtures of solid nanoparticles and block copolymers can yield complex, highly ordered composites for next generation catalysts, selective membranes, and photonic band gap materials $(1-3)$. The specific morphology and hence the utility of these materials depends on the copolymer architecture and on such parameters as the size and volume fraction of the particles. Here, we present a method for calculating the morphology and thermodynamic behavior of copolymer-particle mixtures without requiring a priori knowledge of the equilibrium structures. The method combines a self-consistent field theory (SCFT) for polymers and a density functional theory (DFT) for particles. The SCFT has been remarkably successful in describing the thermodynamics of pure polymer systems (4), whereas DFTs capture particle ordering and phase behavior in colloidal systems $(5,6)$. Applied to a diblock-particle mixture, this technique identifies new self-assembled (SA) morphologies, where both particles and polymers spontaneously order into a mesoscopically regular pattern. We thus delineate conditions where the chains drive particles to self-assemble into continuous "nanowires" or "nanosheets." The method can also be applied to composites involving other copolymer architectures (triblocks, multiblocks, combs, stars) or blends of different polymers.

Our model system consists of a mixture of molten $\mathrm{AB}$ diblock copolymers and solid spherical particles. All particles have the same radius $R$. Each diblock consists of $N$ segments, each of a volume $\rho_{0}{ }^{-1}$. The fraction of A segments per chain is denoted by $f$.

${ }^{1}$ Chemical Engineering Department, University of Pittsburgh, Pittsburgh, PA 15261, USA. ${ }^{2}$ Polymer Science Centre, University of Reading, Whiteknights, Reading RG6 6AF, UK.

*Present address: Dow Chemical Company, Building 1702, Midland, MI 48674, USA.

†To whom correspondence should be addressed. Email: balazs1@engrng.pitt.edu
The enthalpic interaction between an A segment and a B segment is described by the dimensionless Flory-Huggins parameter, $\chi_{\mathrm{AB}}$. As a function of $\left(\chi_{\mathrm{AB}} N\right)$ and $f$, a pure diblock melt can form spatially periodic microstructures with lamellar, cylindrical, spherical, or more complicated phases.

In SCF theory, many-body interactions between differing segments are replaced by the interaction of each segment with the average field created by the other segments. Here, $w_{\mathrm{A}}(\mathbf{r})$ is the value at a point $\mathbf{r}$ of the mean field felt by the A segments, $w_{\mathrm{B}}(\mathbf{r})$ denotes the field for $\mathrm{B}$ segments, and $w_{\mathrm{p}}(\mathbf{r})$ represents the field for particles. Using this approach, the free energy (7) for our system is given by

$$
F=F_{\mathrm{e}}+F_{\mathrm{d}}+F_{\mathrm{p}}
$$

The first term, $F_{\mathrm{e}}$, details the enthalpic interactions in the system:

$$
\begin{aligned}
& F_{\mathrm{e}}=\frac{1}{V} \int d \mathbf{r}\left[\chi_{\mathrm{AB}} N \varphi_{\mathrm{A}}(\mathbf{r}) \varphi_{\mathrm{B}}(\mathbf{r})\right. \\
& \left.+\chi_{\mathrm{BP}} N \varphi_{\mathrm{B}}(\mathbf{r}) \varphi_{\mathrm{p}}(\mathbf{r})+\chi_{\mathrm{AP}} N \varphi_{\mathrm{A}}(\mathbf{r}) \varphi_{\mathrm{p}}(\mathbf{r})\right]
\end{aligned}
$$

where $V$ is the volume of the system, $\chi_{\mathrm{AP}}$ and $\chi_{\mathrm{BP}}$ are the interaction parameters between the respective segments and particles, and $\varphi_{\mathrm{A}}(\mathbf{r}), \varphi_{\mathrm{B}}(\mathbf{r})$, and $\varphi_{\mathrm{p}}(\mathbf{r})$ are the dimensionless concentrations of A segments, B segments, and particles, respectively. The diblock entropic free energy $F_{\mathrm{d}}$ is adapted from (4):

$$
\begin{aligned}
F_{\mathrm{d}} & =\left(1-\phi_{\mathrm{p}}\right) \ln \left[\frac{V\left(1-\phi_{\mathrm{p}}\right)}{Q_{\mathrm{d}}}\right] \\
& -\frac{1}{V} \int d \mathbf{r}\left[w_{\mathrm{A}}(\mathbf{r}) \varphi_{\mathrm{A}}(\mathbf{r})+\mathrm{w}_{\mathrm{B}}(\mathbf{r}) \varphi_{\mathrm{B}}(\mathbf{r})\right]
\end{aligned}
$$

where $Q_{\mathrm{d}}$ is the partition function of a single diblock subject to the fields $w_{\mathrm{A}}(\mathbf{r})$ and $w_{\mathrm{B}}(\mathbf{r})$. The overall volume fraction of particles is 


\section{REPOR T S}

given by $\phi_{\mathrm{p}}$, and the prefactor $1-\phi_{\mathrm{p}}$ is the volume fraction of the diblocks. Finally, the particle entropic free energy is given by

$$
\begin{gathered}
F_{\mathrm{p}}=\frac{\phi_{\mathrm{p}}}{\alpha} \ln \left(\frac{V \phi_{\mathrm{p}}}{Q_{\mathrm{p}} \alpha}\right)-\frac{1}{V} \int d \mathbf{r} w_{\mathrm{p}}(\mathbf{r}) \rho_{\mathrm{p}}(\mathbf{r}) \\
+\frac{1}{V} \int d \mathbf{r} \rho_{\mathrm{p}}(\mathbf{r}) \Psi_{\mathrm{hs}}\left[\bar{\varphi}_{\mathrm{p}}(\mathbf{r})\right]
\end{gathered}
$$

where $Q_{\mathrm{p}}$ is the partition function of a single particle subject to the field $w_{\mathrm{p}}(\mathbf{r})$. The local particle volume fraction, $\varphi_{\mathrm{p}}(\mathbf{r})$, is related to the dimensionless center of mass distribution, $\rho_{\mathrm{p}}(\mathbf{r})$, by

$$
\varphi_{\mathrm{p}}(\mathbf{r})=\frac{4 \alpha}{3 \pi R^{3}} \int_{\left|\mathbf{r}^{\prime}\right|<R} \mathbf{d \mathbf { r } ^ { \prime }} \boldsymbol{\rho}_{\mathrm{p}}\left(\mathbf{r}+\mathbf{r}^{\prime}\right)
$$

The parameter $\alpha$ denotes the particle-todiblock volume ratio

$$
\alpha=\frac{4 \pi R^{3} \rho_{0}}{3 N}=\frac{4 \pi}{3}\left(\frac{R}{R_{0}}\right)^{3} \bar{N}^{1 / 2}
$$

where $R_{0} \equiv a N^{1 / 2}$ is the natural size of the polymer, $a$ is the statistical segment length (assumed to be the same for both A and B segments), and $\bar{N} \equiv a^{6} \rho^{2} N$ is the invariant polymerization index. The last term of $F_{\mathrm{p}}$ describes the excess (non-ideal) steric free energy of the particles through the DFT derived by Tarazona $(5,8)$. In particular, $\Psi_{\mathrm{hs}}$ is the Carnahan-Starling function (9) for the excess free energy of a hard-sphere fluid, evaluated with the "weighted" (locally averaged) particle density, $\bar{\varphi}_{\mathrm{p}}(\mathbf{r})$. This form of the DFT can be readily extended to ellipsoidal particles (6). Thus, our model can be generalized to mixtures of copolymers and rods or platelets. By using the same method to compare spherical and ellipsoidal particles, we can determine the effects of particle shape on the self-assembly process and equilibrium structure of the system.

In the mean-field approximation, $w_{\mathrm{A}}(\mathbf{r})$, $w_{\mathrm{B}}(\mathbf{r})$, and $w_{\mathrm{p}}(\mathbf{r})$ are determined by locating saddle points in the free energy functional (Eq. 1) subject to the incompressibility constraint

$$
\varphi_{\mathrm{A}}(\mathbf{r})+\varphi_{\mathrm{B}}(\mathbf{r})+\varphi_{\mathrm{p}}(\mathbf{r})=1
$$

This yields a system of equations that is solved numerically and self-consistently to give possible equilibrium solutions. To obtain these solutions, we implement the combinatorial screening technique of Drolet and Fredrickson $(10,11)$. The advantage of this technique is that it requires no a priori knowledge of the equilibrium morphology; this is particularly important in our studies because the diblock-particle system can exhibit structures that are as yet unknown. We make an initial random guess for the fields and calculate all the densities and the free energy at each step; the fields are then recalculated and the entire process is repeated until changes in the diblock densities at each step become sufficiently small. In addition, we also minimize our free energy with respect to the size of the simulation box, as proposed by Bohbot-Raviv and Wang (12).

The system is specified by seven parameters: $\chi_{\mathrm{AB}} N, \chi_{\mathrm{AP}} N, \chi_{\mathrm{BP}} N, \bar{N}, f, R$, and $\phi_{\mathrm{p}}$. We fix $\chi_{\mathrm{AB}} N=20$ because we are only concerned that the value be sufficiently large to drive the microphase separation of the diblocks. To model an affinity between the particles and one of the blocks (A), we treat the particles as chemically identical to $\mathrm{A}$, setting $\chi_{\mathrm{AP}}=0$ and $\chi_{\mathrm{BP}}=\chi_{\mathrm{BA}}=\chi_{\mathrm{AB}}$. This causes the particles to be preferentially localized in the A domains. For $\bar{N}$, we assign a typical value of 1000 .

The calculations are first performed in one dimension, allowing for composition variations in only one direction. Thus, we can resolve lamellar morphologies and investigate the distribution of particles within the system. The particle radius is initially set at $R=0.3 R_{0}$. The results of varying the particle volume fraction, $\phi_{\mathrm{p}}$, at $f=0.35$ are shown in Fig. 1, A and B. With $\phi_{\mathrm{p}}=0.15$, Fig. 1A reveals a new SA morphology where the entire composite displays spatial ordering. The A density distribution along the lateral direction, $\varphi_{\mathrm{A}}(x)$, shows a noticeable "crater" in the center of the A lamellae, indicating an exclusion of A chains from this region. The profiles for the particles, $\varphi_{\mathrm{p}}(x)$, and their centers, $\rho_{\mathrm{p}}(x)$, show that the spheres are localized within this cavity. The entire system has organized into a well-ordered "coreshell" structure, with the particles forming nanosheets within the polymer matrix. When the particle volume fraction is lowered to $\phi_{\mathrm{p}}=0.03$ for the same system (Fig. 1B), there are too few particles to form a solid core and thereby exclude the A blocks from the central region. Thus, the composite cannot form the highly ordered SA phase.

Figure $1 \mathrm{C}$ shows a system with smaller particles $\left(R=0.20 R_{0}\right)$ and a volume fraction of $\phi_{\mathrm{p}}=0.15$. Instead of the SA phase seen in Fig. 1A, we now observe a new "edge-assembled" morphology, with the particles forced nearer the $\mathrm{AB}$ interface (as shown by the particle center distribution). The A-block

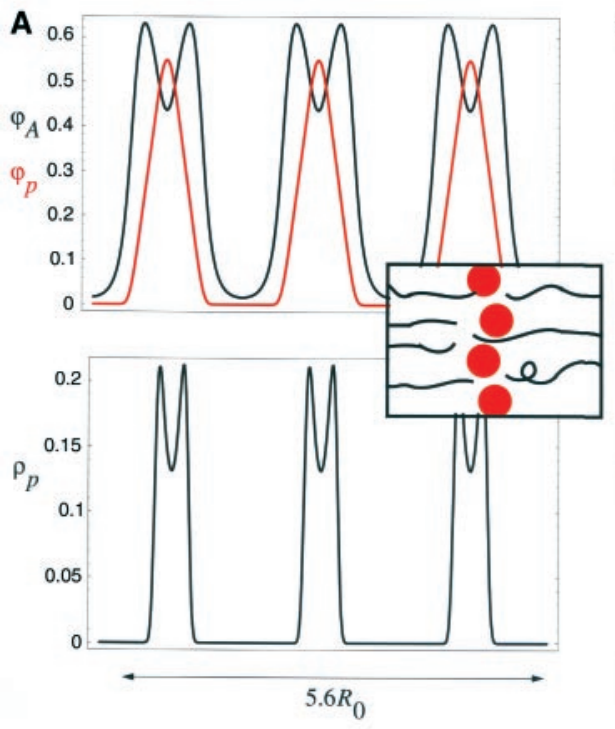

Fig. 1. Concentration profile of diblock-particle systems $\left[\varphi_{A}(x)\right.$, density distribution of A blocks; $\varphi_{p}(x)$ and $\rho_{p}(x)$, distributions of particles and particle centers, respectively]. (A) Large particle $\left(R=0.3 R_{0}\right)$, particle volume fraction $\phi_{\mathrm{p}}=0.15$. (B) Large particle $\left(R=0.3 R_{\mathrm{o}}\right), \phi_{\mathrm{p}}=0.03$. (C) Small particle
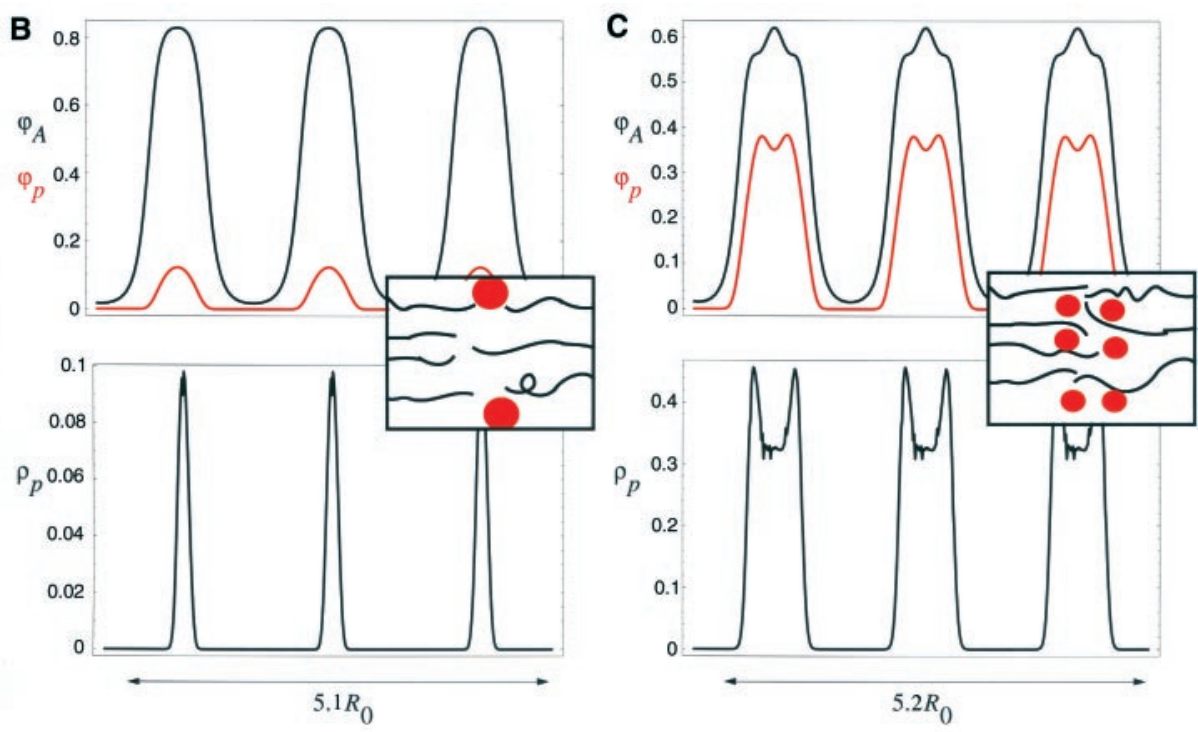

$\left(R=0.2 R_{\mathrm{o}}\right), \phi_{\mathrm{p}}=0.15$. B blocks are not depicted. Three periods are shown to better illustrate the morphologies. Sketches showing the structure of each system accompany the profiles. 
R E P O R T S

concentration is increased in the center of the A domain. Thus, our model indicates that variations in the size and volume fraction of particles can be exploited to control the particle distribution inside the composite.

These results are in qualitative agreement with recent experimental studies by LauterPasyuk et al. (13-15) on thin films of symmetric polystyrene-polybutylmethacrylate (PSPBMA) diblock copolymers with incorporated nanoparticles. Large nanoparticles $(6 \mathrm{~nm})$ localize in the center of the lamellar PS domains while smaller particles $(4 \mathrm{~nm})$ concentrate at the PS-PBMA interfaces, in agreement with Fig. 1, $\mathrm{A}$ and $\mathrm{C}$. Our results also agree with recent Monte Carlo simulations and scaling theory by Huh et al. (16) on melts of diblocks and particles in three dimensions; the study predicts the presence of a SA lamellar structure (Fig. 1A) at comparable values of $R, \phi_{\mathrm{p}}$, and $f$.

The differences among the three structures in Fig. 1 can be understood by considering the relative entropic contributions of the A blocks and particles. For small volume fractions of large particles, the A subchains must stretch to get around the dispersed spheres, incurring a loss in conformational entropy. As $\phi_{\mathrm{p}}$ is increased, the stretching of the A segments is reduced by the segregation of the particles into a central core. (In the SA phase, this free energy gain for the polymers offsets the loss of translational entropy for the particles.) On the other hand, for the same volume fractions of small particles, the stretching required by the polymers to circumvent the spheres is less significant. Hence, it is the translational entropy of the particles that dominates the behavior of the system. As more small particles are added to the system, the spheres are more uniformly dispersed and the entropic free energy per A block increases.

Our theory allows us to break the free energy into components a posteriori, and to test this hypothesis. In Fig. 2, the A-block conformational entropic contribution to the free energy per polymer chain is shown as a function of the particle volume fraction $\phi_{\mathrm{p}}$. For larger particles, the entropic free energy of the A block indeed decreases with increasing $\phi_{\mathrm{p}}$; for smaller particles, this effect disappears.

The previous calculations were performed in one dimension. However, the addition of particles can, in principle, cause the formation of more complex structures, requiring a two- or even three-dimensional description. To check the stability of the lamellar morphologies and confirm the formation of particle nanosheets, we performed a two-dimensional study of the system described in Fig. 1C (17). The results show an increase in the central A-block concentration, as expected from the one-dimensional calculations for the edge-assembled lamellar phase (Fig. 3, A and B), as well as the effects of crystallization within the A domain as

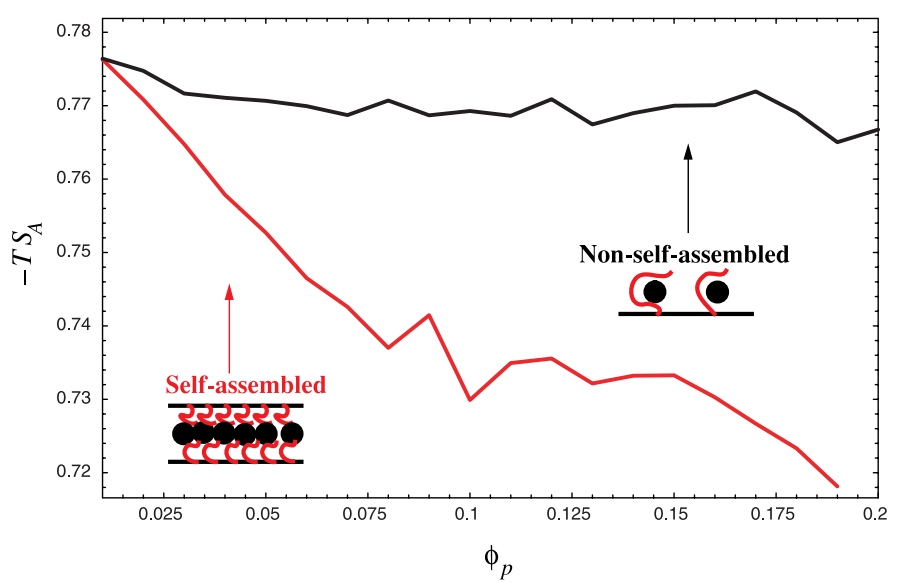

Fig. 2. A-block entropic free energy contribution $-T S_{A}$ (where $S_{A}$ is the conformational entropy of the A block) per polymer chain, for large particles $(R=$ $0.3 R_{0}$, red curve) and small particles $(R=$ $0.15 R_{0}$, black curve), as a function of $\phi_{\mathrm{p}}$.
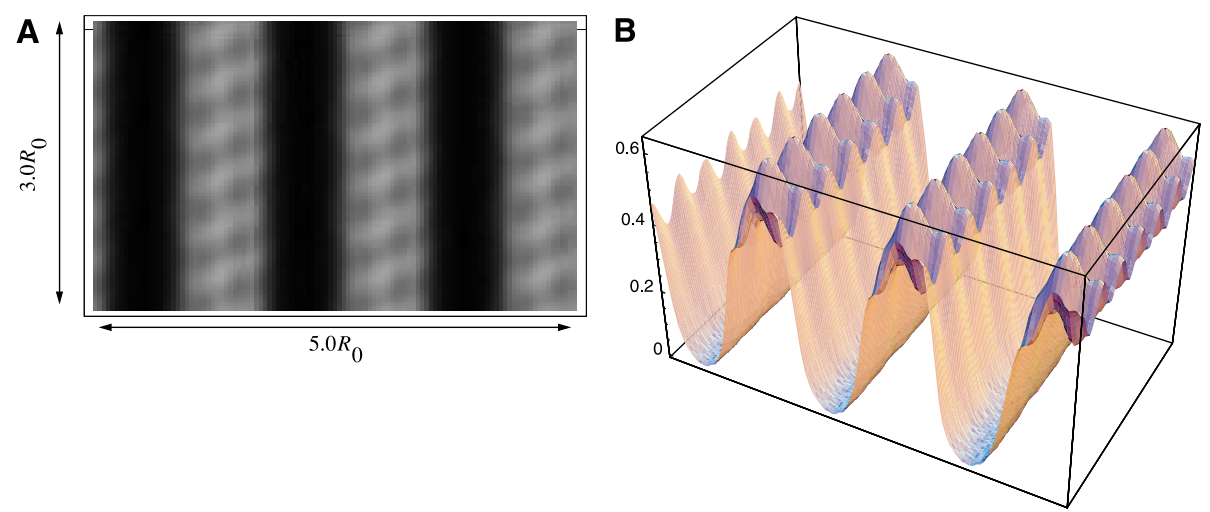

C

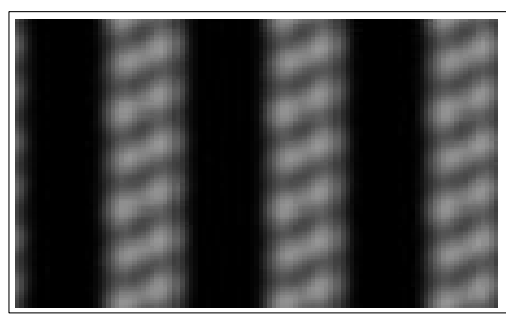

D
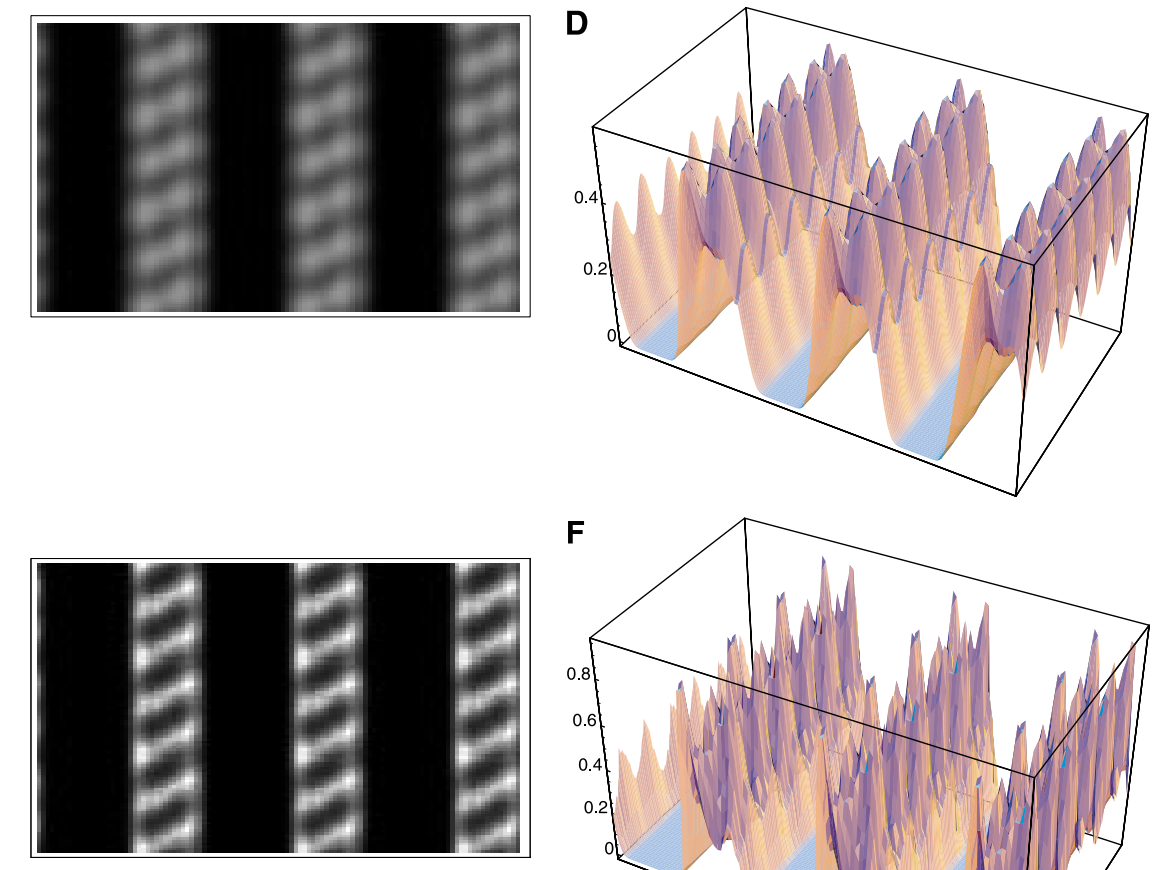

$\mathbf{F}$

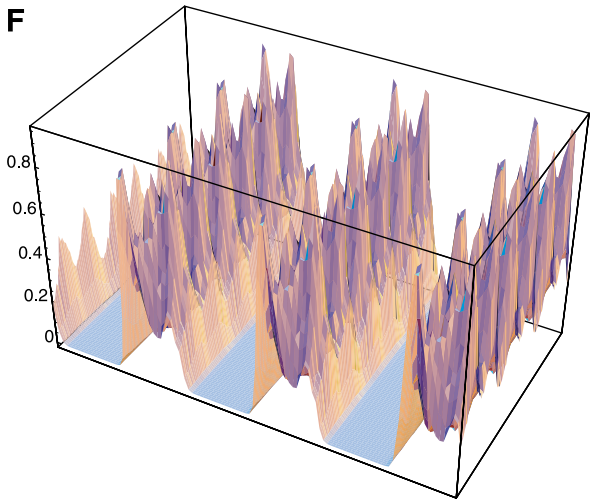

Fig. 3. Representations of a diblock-particle system $\left(R=0.20 R_{0}, \phi_{\mathrm{p}}=0.20\right)$ in two dimensions. Density plots and surface plots are given for the A-block distribution (A and $\mathbf{B})$, the particle distribution (C and $\mathbf{D})$, and the particle center distribution (E and $\mathbf{F}$ ). 


\title{
R E P O R T S
}

the particles localize to fixed positions (Fig. 3, $\mathrm{C}$ to $\mathrm{F}$ ). This cannot be observed in the onedimensional case. Two-dimensional calculations for the large-particle case are not depicted, but they also confirm the morphology shown in one dimension by Fig. 1A. Additionally, crystallization effects are seen in the two-dimensional calculation, just as in the small-particle case. We note, however, that the detailed crystal arrangement may change in three dimensions.

The method introduced here allows us to capture the synergistic interactions that occur in self-assembling mixtures of flexible copolymers and hard particles. It reveals how enthalpic and entropic effects in multiphase media contribute to the observed morphologies and, as in our case, can lead to entropically driven particle crystallization. The versatility of this theory allows us to study the role of copolymer architecture, particle shape, and composite composition, all without making a priori assumptions about the structure of the system. Furthermore, we can compare the free energies of competing structures and construct equilibrium phase diagrams. Overall, the technique can offer valuable assistance in exploring the rich variety of mesostructures that can occur in polymer-particle mixtures and in designing hybrid materials with the desired morphology.

References and Notes

1. M. Templin et al., Science 278, 1795 (1997).

2. D. Zhao et al., Science 279, 548 (1998)

3. S. Stupp, P. V. Braun, Science 277, 1242 (1997).

4. M. W. Matsen, F. S. Bates, Macromolecules 29, 1091 (1996) and references therein.

5. P. Tarazona, Mol. Phys. 52, 81 (1984).

6. G. J. Vroege, H. N. W. Lekkerkerker, Rep. Prog. Phys. 55, 1241 (1992) and references therein.

7. The "free energy" discussed here is actually a dimensionless free energy density: $F \rightarrow N F / \rho_{\mathrm{o}} k_{\mathrm{B}} T V$, where $k_{\mathrm{B}}$ is the Boltzmann constant and $T$ is absolute temperature.

8. The Tarazona DFT was selected because it is a simple and physical approach specifically developed to reproduce the liquid-solid transition in hard spheres. Some DFTs more accurately describe the liquid equation of state but fail to predict the liquid-solid transition; other approaches are more computationally intensive.

9. N. F. Carnahan, K. E. Starling, J. Chem. Phys. 51, 635 (1969).

10. F. Drolet, G. H. Fredrickson, Phys. Rev. Lett. 83, 4317 (1999).

11. This approach may also be better suited to examining morphologies that lose their symmetry on the scale of the particle size. See the "crystallization" effect in Fig. 3 as an example.

12. Y. Bohbot-Raviv, Z.-G. Wang, Phys. Rev. Lett. 85, 3428 (2000).

13. V. Lauter-Pasyuk et al., Physica B 241, 1092 (1997).

14. V. Lauter-Pasyuk et al., Physica B 248, 243 (1998).

15. B. Hamdoun et al., J. Phys. (France) I/ 6, 493 (1996).

16. J. Huh, V. V. Ginzburg, A. C. Balazs, Macromolecules 33, 8085 (2000).

17. The volume fraction has been increased from 0.15 to 0.20 so as to better illustrate "crystallization" effects. Aside from such effects, the morphology is found to be the same as at 0.15 .

18. Supported by the U.S. Department of Energy, NSF, the Army Research Office, and the Engineering and Physical Sciences Research Council (UK).

12 March 2001; accepted 24 May 2001

\section{Diapiric Flow at Subduction Zones: A Recipe for Rapid Transport}

\author{
Paul S. Hall* and Chris Kincaid
}

\begin{abstract}
Recent geochemical studies of uranium-thorium series disequilibrium in rocks from subduction zones require magmas to be transported through the mantle from just above the subducting slab to the surface in as little as $\sim 30,000$ years. We present a series of laboratory experiments that investigate the characteristic time scales and flow patterns of the diapiric upwelling model of subduction zone magmatism. Results indicate that the interaction between buoyantly upwelling diapirs and subduction-induced flow in the mantle creates a network of low-density, low-viscosity conduits through which buoyant flow is rapid, yielding transport times commensurate with those indicated by uranium-thorium studies.
\end{abstract}

Volcanism associated with the subduction of oceanic lithosphere is the primary source of new continental crust and contributes to the chemical differentiation of the crust-mantle system. The mechanism of melt generation at subduction zones and transport to the surface remains uncertain. Proposed models include diapiric flow of a hydrous and/or molten component (1-5), porous flow of magma (6), and melt propagation through fractures $(7,8)$. Constraints on the melting process at subduction zones come from both geochemical $(2,3$, $9-13)$ and geophysical $(14,15)$ studies. In particular, excesses of ${ }^{238} \mathrm{U}$ relative to ${ }^{230} \mathrm{Th}$ observed in rocks from the Mariana (13) and Tonga-Kermadec (11) arcs require the time between the fractionation event responsible for the disequilibrium (i.e., slab dehydration) and eruption of the magma to be as short as $\sim 30$ thousand years (ky). Here we report the results of a series of laboratory experiments conducted to evaluate the characteristic time scales and morphologies of the diapiric flow model.

Conceptual models of mantle diapirism at subduction zones (1-5) invoke the growth of gravitational instabilities within a thin buoyant layer at the upper surface of the subducting slab to explain arc volcanism (Fig. 1A). Individual instabilities detach from the layer and rise through the mantle wedge as diapirs along trajectories determined by the balance between buoyancy forces and drag from subduction-induced motion in the mantle wedge. The diapir model can explain some geophysical observations of magmatism at subduction zones, including the observed regularity of spacing between discrete volcanic centers along $\operatorname{arcs}(d=70 \mathrm{~km})$ and the presence and character of secondary, or transverse, volca-

Graduate School of Oceanography, University of Rhode Island, Narragansett, RI 02882, USA.

*To whom correspondence should be addressed: Email: phall@gso.uri.edu nism at many arcs $(1,16,17)$. It also accounts for many of the petrologic characteristics of arc rocks $(3,4)$. Previous laboratory studies of the diapir model were concerned with the physics of instability growth from a line of buoyant, low-viscosity fluid embedded within a stagnant, viscous fluid and focused on spatial variations in the resulting flow $(1,17$, 18). Our work extends these earlier experiments by including the effect of flow in the viscous fluid and focusing on temporal variations in the patterns of buoyant flow.

Results are presented from a series of 10 laboratory experiments in which we simulated subduction-driven (matrix) flow with a concentrated sucrose solution and flow of buoyant (hydrated or partially molten) mantle with a dyed alcohol solution (Fig. 1B). The buoyant fluid is introduced from a point source, representing what would be a single site of instability growth from a linear buoyancy source [the distribution of instabilities along a linear buoyancy source was previously investigated and yielded a regular spacing of $\sim 70 \mathrm{~km}$ between instabilities (1)]. This parameterization of the buoyancy source is necessary in investigating the temporal evolution of the flow because of the experimental difficulties inherent in maintaining a constant linear source of compositionally buoyant fluid. The flux of buoyant fluid and the velocity of the subducting plate are varied to investigate a range in the relative strength of buoyant and forced flow. Laboratory and mantle length scales are related by assuming that the depth of the buoyancy source in the lab apparatus, $D(17 \mathrm{~cm})$, corresponds to the uniform depth of the Wadati-Benioff zone observed directly beneath island arcs $(125 \mathrm{~km})$. With this fixed length scale, the Mylar velocity can be scaled to a subduction velocity in the mantle through the dimensionless ratio of buoyant flow velocity (from Stokes' law for a spherical body) to subduction velocity $\left(U_{0}\right)$ given by 